\title{
Lace: non-blocking split deque for work-stealing
}

\author{
Tom van Dijk ${ }^{\star}$ and Jaco van de Pol \\ Formal Methods and Tools, Dept. of EEMCS, University of Twente \\ P.O.-box 217, 7500 AE Enschede, The Netherlands \\ \{t.vandijk, vdpol\}@cs.utwente.nl
}

\begin{abstract}
Work-stealing is an efficient method to implement load balancing in fine-grained task parallelism. Typically, concurrent deques are used for this purpose. A disadvantage of many concurrent deques is that they require expensive memory fences for local deque operations.

In this paper, we propose a new non-blocking work-stealing deque based on the split task queue. Our design uses a dynamic split point between the shared and the private portions of the deque, and only requires memory fences when shrinking the shared portion.

We present Lace, an implementation of work-stealing based on this deque, with an interface similar to the work-stealing library Wool, and an evaluation of Lace based on several common benchmarks. We also implement a recent approach using private deques in Lace. We show that the split deque and the private deque in Lace have similar low overhead and high scalability as Wool.
\end{abstract}

Keywords: work-stealing, task-based parallelism, dynamic load balancing, lock-free algorithm, non-blocking deque

\section{Introduction}

\subsection{Task-based parallelism}

In recent years, the importance of using parallelism to improve the performance of software has become self-evident, especially given the availability of multicore shared-memory systems and the physical limits of processor speeds. Frameworks like Cilk $[3,9]$ and Wool $[7,8]$ allow writing parallel programs in a style similar to sequential programs [1].

In task-based parallelism, a computation is divided into small tasks. Each task only depends on the results of its own immediate subtasks for its execution. Multiple independent subtasks can be executed in parallel. Especially recursive algorithms are easily parallelized.

Cilk, Wool, and similar task-based parallel frameworks use keywords spawn and sync to expose parallelism. The spawn keyword creates a new task. The sync keyword matches with the last unmatched spawn, i.e., operating as if spawned tasks are stored on a stack. It waits until that task is completed and

\footnotetext{
* The first author is supported by the NWO project MaDriD, grant nr. 612.001.101
} 
in Cilk in 1996 [3]. One improvement of the original Cilk algorithm is the THE protocol in Cilk-5 [9], which eliminates acquiring the lock in push and in most executions of pop, but every steal still requires locking.

The first non-blocking work-stealing deque is the ABP algorithm, which uses a fixed-size array that might overflow [2]. Two unbounded non-blocking deques were proposed, the deque by Hendler et al. based on linked lists of small arrays [10], and the Chase-Lev deque that uses dynamic circular arrays [5].

In weak memory models that allow reordering loads before stores, most deques that allow any spawned task to be stolen require a memory fence in every pop operation. Memory fences are expensive. For example, the THE protocol spends half of its execution time in the memory fence [9].

Several approaches alleviate this problem. The split task queue by Dinan et al. [6], designed for clusters of multiprocessor computers, allows lock-free local access to a private portion of the queue and can transfer work between the public and private portions of the queue without copying tasks. Thieves synchronize using a lock and the local process only needs to take the lock when transferring work from the public portion to the private portion of the queue. Michael et al. propose relaxed semantics for work-stealing: inserted tasks are executed at least once instead of exactly once, to avoid requiring memory fences and atomic instructions [12]. In the work scheduler Wool [7], originally only the first $N$ tasks in the deque can be stolen, where $N$ is determined by a parameter at startup. Only executing pop on stealable tasks requires a memory fence. In a later version, the number of stealable tasks is dynamically updated [8].

In some work-stealing algorithms, shared deques are replaced by private deques, and work is explicitly communicated using a message-passing approach. Recently, Acar et al. proposed two algorithms for work-stealing using private deques [1]. See further [1] for an overview of other work with private deques.

Tasks are often stored as pointers that are removed from the deque when the task is stolen $[9,2,10,5]$. To virtually eliminate the overhead of task creation for tasks that are never stolen, Faxén proposed a direct task stack, storing tasks instead of pointers in the work queue, implemented in Wool $[7,8]$. Rather than synchronizing with thieves on the metadata of the queue (e.g. variables top and bot in the ABP algorithm), Wool synchronizes on the individual task descriptors, using locks when synchronizing with potential thieves, similar to the THE protocol. Sundell and Tsigas presented a lock-free version of Wool $[15,8]$, which still synchronizes on the individual task descriptors.

\subsection{Contributions}

Acar et al. write that concurrent deques suffer from two limitations: 1) local deque operations (mainly pop) require expensive memory fences in modern weakmemory architectures; 2) they can be very difficult to extend to support various optimizations, especially steal-multiple extensions [1]. They lift both limitations using private deques. Wool reduces the first limitation for concurrent deques by using a dynamic number of stealable tasks, but is difficult to extend for steal-multiple strategies, since tasks must be stolen individually. 
We present a work-stealing algorithm that eliminates these limitations using concurrent deques, by combining a non-blocking variant of the split task queue [6] with direct task stealing from Wool $[7,8]$. This algorithm splits the deque into a shared portion and a private portion. The split point between these portions is modified in a non-blocking manner.

We present an implementation of this algorithm in a C library called Lace ${ }^{1}$, which has the same interface as Wool. We evaluate the performance of Lace using several benchmarks, including standard Cilk benchmarks and the UTS benchmark [13]. We compare our algorithm with Wool and with an implementation of the receiver-initiated private deque algorithm [1] in the Lace framework. Our experiments show that our algorithm is competitive with both Wool and the private deque algorithm, while lifting both limitations described in [1]. Compared to the private deque algorithm, our algorithm allows stealing of all tasks in the shared deque without cooperation of the owner, while the private deque algorithm requires cooperation of the owner for every steal transaction.

\section{Preliminaries}

We assume a shared memory system with the x86 memory model. The x 86 memory model is not sequentially consistent, but allows reordering loads before stores. Memory writes are buffered before reaching the memory, hence reads can occur before preceding memory writes are globally visible. Memory fences flush the write buffer before reading from memory. Apart from memory fences, we use the atomic memory operation compare_and_swap (cas) to ensure safety. The cas operation atomically compares a value in memory to an expected value and modifies it only if the values match. We use cas to ensure that exactly one worker performs a transition.

We assume that the system consists of one or more processor chips and one or more memory chips, connected using an interconnection network, for example in Non-Uniform Memory Access (NUMA) shared-memory systems. We also assume that data on this interconnection network is transferred in blocks called cachelines, which are typically 64 bytes long.

\section{Algorithm}

\subsection{Design considerations}

To obtain a low execution time when performing work-stealing with all available workers, we aim at low overhead compared to purely sequential programs and good scalability with increasing worker count. Memory fences and cas operations increase the overhead compared to purely sequential programs. Some memory fences are unavoidable, since thieves may steal a task while the owner is retrieving it. By splitting the deque into a shared deque and a private deque (see Figure 2),

\footnotetext{
${ }^{1}$ Lace is available at http://fmt.ewi.utwente.nl/tools/lace/.
} 

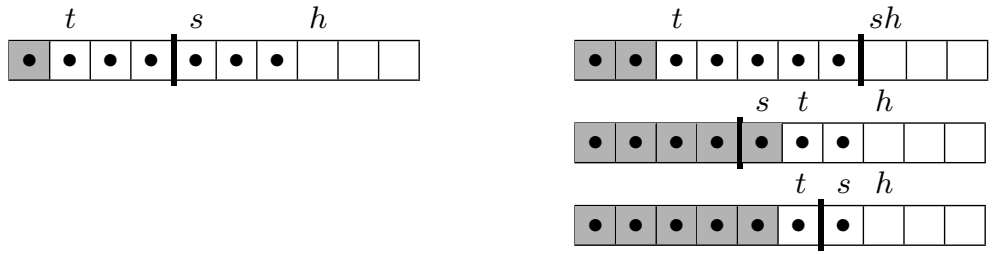

Figure 2. The split deque, with tail $t$, split point $s$ and head $h$. A task at position $x$ is stolen if $x<t$. It is shared if $x<s$, and private otherwise. Of the 7 tasks in this example, 4 are shared and 1 is stolen.
Figure 3. The owner shrinks the shared portion of the deque, but thieves may have stolen tasks beyond the new split point. The owner detects this and updates the split point to its final position.

we only need a memory fence when shrinking the shared deque, to detect the scenario of Figure 3. Also, cas operations are only needed to coordinate stealing.

The deque is described using variables tail, split and head, which are indices in the task array. To steal work, thieves only require knowledge of tail and split, and only need to modify tail. The owner uses head and o_split (a private copy of split) to operate on the private deque. The owner only accesses tail and split when changing the split point.

Thieves are not allowed to change the split point, since this would force a memory fence on every execution of pop. Instead, thieves set a shared flag splitreq on a dedicated cacheline when there are no more unstolen shared tasks. Since splitreq is checked at every execution of pop and push, it should always be in the processor cache of the owner, and no traffic on the interconnect network is expected until the flag is set. There is no other communication between the owner and the thieves, except when tasks are stolen soon after their creation, or when the owner is waiting for an unfinished stolen task.

If the owner determines that all tasks have been stolen, it sets a flag allstolen (and a private copy o_allstolen). Thieves check allstolen first before attempting to steal tasks, which results in a small performance gain. When the owner already knows that all tasks are stolen, it does not need to shrink the shared deque until new tasks are added.

Similar to the direct task stack in Wool, the deque contains fixed-size task descriptors, rather than pointers to task descriptors stored elsewhere. Stolen tasks remain in the deque. The result of a stolen task is written to the task descriptor. This reduces the task-creation overhead of making work available for stealing, which is important since most tasks are never stolen. Another advantage is that the cachelines accessed by a thief are limited to those containing the task descriptor and the variables tail, split and (rarely) splitreq, while in designs that use pointers, there is at least one additional accessed cacheline. If task descriptors are properly aligned and fit into one cacheline, then thieves only access two cachelines per successful steal. Also, in a pointer-based design, there are many pointers per cacheline, which can increase contention on that cacheline. 
Method pop determines whether the last task is stolen. This is the case when allstolen is set, or when all tasks are shared (i.e., o_split = head) and the method shrink_shared reports that all tasks are stolen. If the last task is stolen, then it remains on the deque. If the last task is not stolen, then head is decreased, and if splitreq is set, pop calls grow_shared.

If the last task is stolen, then pop_stolen is called after the stolen task is completed (see Figure 1). Leapfrogging may have changed the state of the deque, therefore allstolen is set again, since the remaining tasks are still stolen.

In grow_shared, the new value of the split point is the ceiling of the average of split and head. Since grow_shared is only called if not allstolen, i.e., split $\leq$ head, the shared deque will always grow and therefore atomic operations or memory fences are not necessary.

Method shrink_shared moves the split point to decrease the size of the shared deque. It then detects whether thieves have stolen tasks beyond the new split point, and if so, it moves the split point again. If all tasks were stolen, then shrink_shared sets allstolen and returns True. It returns False otherwise. Since shrink_shared is called by the owner only if split = head, line 43 really checks whether tail = head, i.e., whether all tasks are stolen. If not, then the split point is moved between tail and split. The memory fence ensures that the new split point is globally visible before reading tail. Once the new split point is globally visible, no tasks can be stolen beyond the new split point. Therefore, we only need to check once whether more tasks are stolen. If at that point all remaining tasks are stolen, then allstolen is set and shrink_shared returns True. If not, then if only some tasks are stolen beyond the new split point, the split point is moved again. Finally, shrink_shared returns False.

\subsection{Extensions}

There are several possible extensions to the work-stealing deque.

Resizing. Our work-stealing deque uses a fixed-size array. Given that virtual memory is several orders of magnitude larger than real memory and the ability of modern operating systems to allocate only used pages, we can avoid overflows by allocating an amount of virtual memory much higher than required. The deque could be extended for resizing, for example using linked lists of arrays, but we feel this is unnecessary in practice.

Steal-multiple strategies. One extension to work-stealing is the policy to steal more than one task at the same time, e.g., stealing half the tasks in the deque, which has been argued to be beneficial in the context of irregular graph applications $[11,6]$. This is easily implemented by modifying line 5 to steal multiple tasks, and executing the stolen tasks in reverse order (last one first). However, in experiments on a single NUMA machine, this did not improve performance.

Other memory models. The algorithm in Figure 4 is designed for the x86 TSO memory model, which only allows reordering loads before stores. Weaker memory models may for example allow reordering stores. Assuming that reordering only takes place on independent operations, we believe no additional memory fences are 
required in Figure 4 to ensure correctness. Memory fences are however required in Figure 1 to ensure that result is set before done.

\section{Evaluation}

We implemented Lace, a C library that provides a work-stealing framework similar to Wool and Cilk. The library creates one POSIX thread (pthread) for each available core. Our implementation is NUMA-aware, i.e., all pthreads are pinned to a NUMA domain and their program stack and the deque structures for each worker are allocated on the same NUMA domain as the worker.

We evaluate Lace using several benchmarks compared to the work-stealing framework Wool [8] using the classic leapfrogging strategy. This version of Wool has a dynamic split point and does not use locking. We compare the performance of Lace and Wool, for two reasons. Our implementation resembles the implementation of Wool, making a comparison easier. Also, [8] and [14] show that Wool is efficient compared to Cilk++, OpenMP and the Intel TBB framework, with a slight advantage for Wool. We also compare our algorithm to the receiver-initiated version of the private deque of Acar et al. [1], using the alternative acquire function, which we implemented in the Lace framework.

\subsection{Benchmarks}

For all benchmarks, we use the smallest possible granularity and do not use sequential cut-off points, since we are interested in measuring the overhead of the work-stealing algorithm. Using a larger granularity and sequential cut-off points may result in better scalability for some benchmarks.

Fibonacci. For a positive integer $N$, calculate the Fibonacci number by calculating the Fibonacci numbers $N-1$ and $N-2$ recursively and add the results. This benchmark generates a skewed task tree and is commonly used to benchmark work-stealing algorithms, since the actual work per task is minimal. Number of tasks: 20,365,011,073 (fib 50).

Queens. For a positive integer $N$, calculate the number of solutions for placing $N$ queens on a $N \times N$ chessboard so that no two queens attack each other. Each task at depth $i$ spawns up to $N$ new tasks, one for every correct board after placing a queen on row $i$. Number of tasks: 171,129,071 (queens 15).

Unbalanced Tree Search. This benchmark is designed by Olivier et al. to evaluate the performance for parallel applications requiring dynamic load balancing. The algorithm uses the SHA-1 algorithm to generate geometric and binomial trees. The generated binomial trees (T3L) have unpredictable subtree sizes and depths and are optimal adversaries for load balancing strategies [13] The geometric tree (T2L) appears to be easy to balance in practice. Number of tasks: 96,793,509 (uts T2L) and 111,345,630 (uts T3L).

Rectangular matrix multiplication. Given $N$, compute the product of two random rectangular $N \times N$ matrices $A$ and $B$. We use the matmul algorithm from the Cilk benchmark set. Number of tasks: 3,595,117 (matmul 4096). 


\begin{tabular}{|l|c|c|c|c|}
\hline \multirow{2}{*}{ Benchmark } & \multicolumn{2}{|c|}{ Lace } & \multicolumn{2}{c|}{ Speedup } \\
\cline { 2 - 5 } & $T_{1}$ & $T_{48}$ & $T_{S} / T_{48}$ & $T_{1} / T_{48}$ \\
\hline fib 50 & 144 & 4.13 & 34.5 & 34.9 \\
uts T2L & 86.0 & 1.81 & 46.1 & 47.4 \\
uts T3L & 44.2 & 2.23 & 18.7 & 19.9 \\
queens 15 & 602 & 12.63 & 42.2 & 47.7 \\
matmul 4096 & 781 & 16.46 & 47.0 & 47.5 \\
\hline \multicolumn{4}{|c|}{ Private deque } \\
\hline fib 50 & 208 & 5.22 & 23.2 & 39.8 \\
uts T2L & 86.1 & 1.83 & 45.7 & 47.0 \\
uts T3L & 44.8 & 2.55 & 17.3 & 17.5 \\
queens 15 & 541 & 11.34 & 43.3 & 47.7 \\
matmul 4096 & 774 & 16.34 & 47.3 & 47.4 \\
\hline
\end{tabular}

\begin{tabular}{|l|c|c|c|c|}
\hline \multirow{2}{*}{ Benchmark } & \multicolumn{2}{|c|}{ Wool } & \multicolumn{2}{c|}{ Speedup } \\
\cline { 2 - 5 } & $T_{1}$ & $T_{48}$ & $T_{S} / T_{48}$ & $T_{1} / T_{48}$ \\
\hline fib 50 & 185 & 4.38 & 34.1 & 42.2 \\
uts T2L & 85.1 & 2.00 & 42.5 & 42.5 \\
uts T3L & 44.3 & 2.12 & 19.4 & 20.9 \\
queens 15 & 539 & 11.23 & 47.5 & 48.0 \\
matmul 4096 & 780 & 16.40 & 47.2 & 47.5 \\
\hline & $T_{S}$ & \multicolumn{3}{|c|}{ Sequential } \\
\hline fib 50 & 149.2 & - & - & - \\
uts T2L & 84.5 & - & - & - \\
uts T3L & 43.11 & - & - & - \\
queens 15 & 533 & - & - & - \\
matmul 4096 & 773 & - & - & - \\
\hline
\end{tabular}

Figure 5. Averages of running times (seconds) for all benchmarks. Speedups are calculated relative to both the time of the sequential version $\left(T_{S}\right)$ and the parallel version with one worker $\left(T_{1}\right)$. Each $T_{48}$ data point is the average of 50 measurements. Each $T_{1} / T_{S}$ data point is the average of 20 measurements.

\subsection{Results}

Our test machine has four twelve-core AMD Opteron 6168 processors. The system has $128 \mathrm{~GB}$ of RAM and runs Scientific Linux 6.0 with kernel version 2.6.32. We considered using less than 48 cores to reduce the effects of operating system interference, but we did not see significant effects in practice. We compiled the benchmarks using gcc 4.7.2 with flag -03.

See Figure 5 for the results of the benchmark set. Each $T_{48}$ data point is the average of 50 measurements. Each $T_{1}$ and $T_{S}$ data point is the average of 20 measurements. This resulted in measurements with three significant digits. In general, Figure 5 shows similar performance for all three algorithms. The three benchmarks uts T2L, queens and matmul are trivial to parallelize and have no extra overhead with 48 workers, i.e., $T_{1} / T_{48} \approx 48$.

Comparing $T_{S}$ and $T_{1}$ for all benchmarks, we see that the overhead of workstealing is small for all three work-stealing algorithms, with the exception of the fib benchmark. For benchmark $\mathrm{fib}$ with our algorithm, $T_{1}<T_{S}$, which appears to be related to compiler optimizations. During implementation, we observed that variation in $T_{1}$ is often related to code generation by the compiler. In some cases, removing unused variables and other minor changes even increased $T_{1}$ by up to $20 \%$. It is therefore difficult to draw strong conclusions regarding the overhead of the algorithms, except that it is small compared to the sequential program.

We measured the runtimes of $\mathrm{fib}$ and uts T3L using 4, 8, 16, 24, 32 and 40 workers to obtain the speedup graph in Figure 6. This graph suggests that the fib benchmark scales well and that similar results may be obtained using a higher number of processors in the future. The scalability of the uts T3L benchmark appears to be limited after 16 workers. We discuss this benchmark below.

We also measured the average number of steals during a parallel run with 48 workers. See Figure 7 . We make a distinction between normal stealing when a 


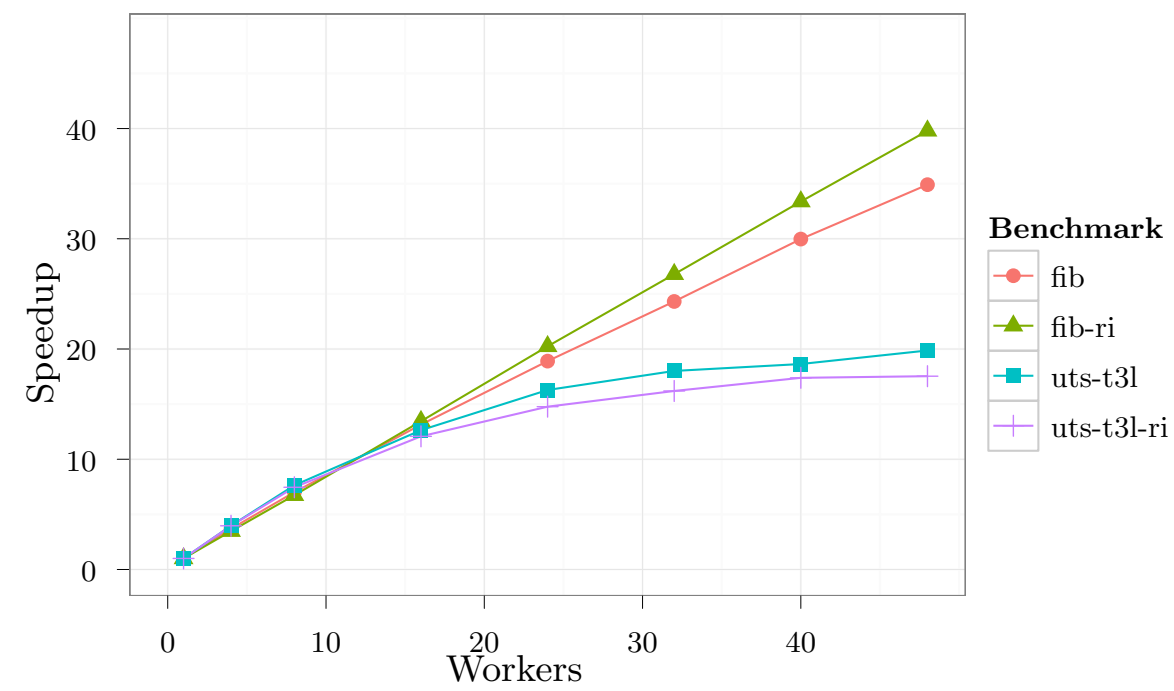

Figure 6. Absolute speedup graph $\left(T_{1} / T_{N}\right)$ of the fib and uts T3L benchmarks using Lace with our algorithm and Lace with the private deque receiver initiated (-ri) algorithm. Each data point is based on the average of 20 measurements.

\begin{tabular}{|l|c|c|c|c|}
\hline Benchm. & \#steals & \#leaps & \#grows & \#shrinks \\
\hline fib 50 & 865 & 50,569 & 70,789 & 97,750 \\
uts T2L & 4,554 & 82,440 & 72,222 & 57,701 \\
uts T3L & 158,566 & $4,443,432$ & $2,173,006$ & 846,509 \\
queens 15 & 1,964 & 6,053 & 5,694 & 6,618 \\
matmul 4096 & 2,492 & 12,456 & 13,081 & 9,911 \\
\hline
\end{tabular}

Figure 7. The average total number of steals, leaps, grows and shrinks over 7 runs with 48 workers.

\begin{tabular}{|l|c|c|c|}
\hline Algo. & $T_{1}$ & $T_{48}$ & $T_{1} / T_{48}$ \\
\hline Lace & 44.26 & 1.154 & 38.3 \\
Private & 44.83 & 1.240 & 36.2 \\
Wool & 44.27 & 1.172 & 37.8 \\
\hline
\end{tabular}

Figure 8. Averages of runtimes (seconds) of uts T3L with transitive leapfrogging (Wool) or random stealing (Lace/Private).

worker is idle, and leapfrogging when a worker is stalled because of unfinished stolen work. We also measured the amount of split point changes by grow_shared and shrink_shared. The number of 'grows' indicates how often thieves set splitreq. The number of 'shrinks' is equal to the number of memory fences. In the uts T3L benchmark, the high number of leaps and split point changes may indicate that the stolen subtrees were relatively small.

\subsection{Extending leapfrogging}

Benchmark uts T3L appears to be a good adversary for all three algorithms. This is partially related to the leapfrogging strategy, which forces workers that wait for the result of stolen tasks to steal from the thief. This strategy can result in chains of thieves waiting for work to trickle down the chain. For example, when worker 2 steals from worker 1 , worker 1 will only steal from worker 2 . If 
worker 3 steals from worker 2 and worker 4 steals from worker 3, new tasks will be generated by worker 4 and stolen by worker 3 first. Worker 3 then generates new work which can be stolen by workers 2 and 4 . Worker 1 only acquires new work if the subtree stolen by worker 4 is large enough. The updated version of Wool [8] implements an extension to leapfrogging, called transitive leapfrogging ${ }^{2}$. Transitive leapfrogging enables workers to steal from the thief of the thief, i.e., still steal subtasks of the original stolen task.

We extended Lace to steal from a random worker whenever the thief has no available work to steal. See Figure 8 for the results of this extension, compared to transitive leapfrogging in Wool. Compared to the results in Figure 5, all benchmarks now have reasonable speedups, improving from a speedup of 20x to a speedup of $36 \mathrm{x}$ with 48 workers.

Our extension has the disadvantage of not guaranteeing the upper bound on the stack size that leapfrogging and transitive leapfrogging does. It is, however, very simple to implement, while resulting in similar performance. We measured the peak stack depth with the uts T3L benchmark for all 48 workers. We observed an increase from a peak stack depth of 6500-12500 tasks with normal leapfrogging to 17000-21000 tasks with the random stealing extension. Since every task descriptor for uts T3L is 64 bytes large (including padding), this strategy required at most 1 extra megabyte per worker for uts T3L. We also observed that the number of 'grows' decreased by $50 \%$.

\section{Conclusion}

In this paper, we presented a new non-blocking split deque for work-stealing. Our design has the advantage that it does not require memory fences for local deque operations, except when reclaiming tasks from the shared portion of the deque. Furthermore, we implemented this deque in a $\mathrm{C}$ library called Lace, which has an interface similar to Wool. This framework has the advantage of a small source code footprint. We also implemented the receiver-initiated version of the private deque algorithm described by Acar et al. in Lace.

Our experiments show that our algorithm is competitive with Wool and with the private deque algorithm. We gain near optimal speedup for several benchmarks, with very limited overhead compared to the sequential program. Extending leapfrogging with random stealing greatly improves scalability for the uts T3L benchmark.

Several open questions remain. When growing the shared deque, the new split point is the average of split and head, and when shrinking the shared deque, the new split point is the average of tail and head. It is unknown whether more optimal strategies exist. A limitation of our approach is that tasks can only be stolen at the tail of the deque. This limits work-stealing strategies. Designs that allow stealing any task may be useful for some applications. Our benchmarks all consist of uniformly small tasks. Benchmarking on larger or

\footnotetext{
${ }^{2}$ This feature is documented in the distribution of Wool version 0.1.5alpha, which is currently available at http://www.sics.se/ kff/wool/.
} 
irregular sized tasks may be disadvantageous for the private deque algorithm, since it requires owner cooperation on every steal. Finally, we performed our experiments on a single NUMA machine. On such machines, communication costs are low compared to distributed systems. It may be interesting to compare the work-stealing algorithms on a cluster of computers using a shared-memory abstraction. Especially steal-multiple strategies may be more beneficial when communication is more expensive.

\section{References}

1. Acar, U.A., Charguéraud, A., Rainey, M.: Scheduling parallel programs by work stealing with private deques. In: PPOPP. pp. 219-228. ACM (2013)

2. Arora, N.S., Blumofe, R.D., Plaxton, C.G.: Thread Scheduling for Multiprogrammed Multiprocessors. Theory Comput. Syst. 34(2), 115-144 (2001)

3. Blumofe, R.D., Joerg, C.F., Kuszmaul, B.C., Leiserson, C.E., Randall, K.H., Zhou, Y.: Cilk: An Efficient Multithreaded Runtime System. J. Parallel Distrib. Comput. 37(1), 55-69 (1996)

4. Blumofe, R.D., Leiserson, C.E.: Scheduling Multithreaded Computations by Work Stealing. In: FOCS. pp. 356-368. IEEE Computer Society (1994)

5. Chase, D., Lev, Y.: Dynamic circular work-stealing deque. In: SPAA. pp. 21-28. ACM (2005)

6. Dinan, J., Larkins, D.B., Sadayappan, P., Krishnamoorthy, S., Nieplocha, J.: Scalable work stealing. In: SC. ACM (2009)

7. Faxén, K.F.: Wool-A work stealing library. SIGARCH Computer Architecture News 36(5), 93-100 (2008)

8. Faxén, K.F.: Efficient Work Stealing for Fine Grained Parallelism. In: ICPP. pp. 313-322. IEEE Computer Society (2010)

9. Frigo, M., Leiserson, C.E., Randall, K.H.: The Implementation of the Cilk-5 Multithreaded Language. In: PLDI. pp. 212-223. ACM (1998)

10. Hendler, D., Lev, Y., Moir, M., Shavit, N.: A dynamic-sized nonblocking work stealing deque. Distributed Computing 18(3), 189-207 (2006)

11. Hendler, D., Shavit, N.: Non-blocking steal-half work queues. In: PODC. pp. 280-289. ACM (2002)

12. Michael, M.M., Vechev, M.T., Saraswat, V.A.: Idempotent work stealing. In: PPOPP. pp. 45-54. ACM (2009)

13. Olivier, S., Huan, J., Liu, J., Prins, J., Dinan, J., Sadayappan, P., Tseng, C.W.: UTS: An Unbalanced Tree Search Benchmark. In: Almási, G., Cascaval, C., Wu, P. (eds.) LCPC. LNCS, vol. 4382, pp. 235-250. Springer (2006)

14. Podobas, A., Brorsson, M.: A comparison of some recent task-based parallel programming models. In: Programmability Issues for Multi-Core Computers,(MULTIPROG'2010), Jan 2010, Pisa (2010)

15. Sundell, H., Tsigas, P.: Brushing the Locks out of the Fur: A Lock-Free Work Stealing Library Based on Wool. In: The Second Swedish Workshop on Multi-Core Computing MCC09. University of Borås. School of Business and Informatics (2009)

16. Wagner, D.B., Calder, B.: Leapfrogging: A portable technique for implementing efficient futures. In: PPOPP. pp. 208-217. ACM (1993) 